\title{
Agile Software Assurance
}

Noura Abbas, Andrew M. Gravell, and Gary B. Wills

School of Electronics and Computer Science, University of Southampton

Southampton, SO17 1BJ, United Kingdom

\{na06r, amg, gbw $\}$ eecs. soton.ac.uk

\section{Research Questions}

Since Agile methods were presented in the 90s, many papers, articles and books have been published about Agile. However, little work focuses on Agile Software Quality. Therefore, the main goal of the $\mathrm{PhD}$ is to study the quality of Agile projects.

We are trying to answer the following research questions:

1. What is the quality of Agile Software, importantly, how we can evaluate the quality of Agile Software?

2. Can Agile assure the quality under time pressure and with unstable requirements?

3. What are the best ways to assure the quality of Agile Software?

4. Different organizations interpret words like Agile and iterative in different ways. Therefore, another question is how do organizations understand these terms?

In order to answer these questions we decided to investigate further Agile Software Assurance, in order to give a clear understanding of the topic, and furthermore, to help people and organizations who work with Agile to produce high quality software.

\section{Related Work and Current Solutions}

There have been many initiatives relating Agile with quality standards such as CMMI and ISO 9000. Although the results of these initiatives vary, they all shared the same idea of modifying Agile methods in order to be accepted from the standards point of view $[1,2,3,5]$.

An interesting point is whether we really need to modify the method in order to achieve the standards. Instead, why not create a standard which can be suitable for the new methodology. The main reason for introducing CMMI was to modify CMM to be more suitable for modern iterative development, interestingly, because few of the modern principles are in conflict with CMM key process areas [4].

\section{Research Approach and Current State}

Interviews and questionnaires constitute the appropriate qualitative and quantitative research methods to collect as much data as possible about Agile in practice. We have started with developing two questionnaires. The first one will be to understand what organizations mean by the term "Agile", and how Agile they are. Then the second questionnaire will be to find out how organizations assure the quality of Agile software products, and what are the reasons behind their success.

G. Concas et al. (Eds.): XP 2007, LNCS 4536, pp. 165-166, 2007.

(C) Springer-Verlag Berlin Heidelberg 2007 
As a start, the questionnaires will be conducted on 10 Agile projects that use Agile in different percentage. In the next stage the questionnaires will be improved and the range of the organizations will be broader.

We have started interviewing people from two organizations and half of dozen individuals who had experience with Agile projects. The interviewees are involved in different roles within the organizations: project managers, developers, testers, architects, and domain experts. The projects were chosen from a range of software development organizations in the UK (where uptake of Agile methods has been slower than in the US). The projects are of different sizes, from a variety of application domains, mainly middleware, web services, electronic design automation, and banking management systems. Our initial findings showed that the final products had different levels of quality. In addition, organizations use different Agile practices to assure the quality: testing, re-factoring, pair programming, recruiting skilled people, and continuous communication with the customer. Importantly, those organizations have different understanding of Agility, and probably this is one of the important reasons behind having "Agile" software with different levels of quality.

The next step will be to analyze the collected data, in order to understand how people used Agile. Furthermore to find out how this usage affects the quality of the final products. The final step will be to bring all the findings together and inform the model which will describe how to improve the quality of Agile projects.

\section{Significance of the Research}

This research will help software development organizations to have deeper understanding of Agile Methods, principles and practices. Moreover, identifying critical links between conditions of success and reasons of failure to achieve high quality Agile software could be a potential research finding. Furthermore, this research will help to evaluate the quality of Agile projects.

From this point of view having a successful quality assurance model for Agile projects will increase the successful use of Agile in both industry and academia.

\section{References}

1. Anderson, D.: Stretching Agile to fit CMMI level 3, Microsoft Corporation (2005)

2. Royce, W.: CMM vs. CMMI: From Conventional to Modern Software Management, Rational Software (2002)

3. Vriens, C.: Certifying for CMM Level 2 and ISO9001 with XP@Scrum. In: Proceedings of the Agile Development Conference (ADC'03) (2003)

4. Nawrocki, J., Jasinski, M., Walter, M., Wojciechowski, A.: Combining Extreme Programming with ISO 9000. In: Shafazand, H., Tjoa, A.M. (eds.) EurAsia-ICT 2002. LNCS, vol. 2510, pp. 786-794. Springer, Heidelberg (2002)

5. Turner, R., Jain, A.: Agile Meets CMMI: Culture Clash or Common Cause? In: Proceedings of the XP/Agile Universe 2002, pp. 153-165. Springer-Verlag, Heidelberg (2002) 